\title{
Korrelation petrophysikalischer und geomechanischer Parameter von Gesteinen des Erzberges
}

\author{
Nina Gegenhuber ${ }^{1}$, Melanie Krüger² und David SeidI $^{1}$ \\ ${ }^{1}$ Lehrstuhl für Subsurface Engineering, Montanuniversität Leoben, Leoben, Österreich \\ ${ }^{2}$ Swietelsky Tunnelbau GmbH \& Co KG, Salzburg, Österreich
}

Eingegangen 16. Oktober 2021; angenommen 11. November 2021; online publiziert 26. November 2021

\begin{abstract}
Zusammenfassung: Die Korrelation von geophysikalischen/petrophysikalischen Parametern mit geotechnischen bietet für die Planung und Erstellung von Untertageinfrastrukturen und Geothermie sowie Erdöl Projekten einen großen Mehrwert. Gerade der interdisziplinäre Ansatz von Geologie, Mineralogie, Geophysik und Geotechnik kann in der Zukunft bei Herausforderungen der Zeit helfen und ist damit unumgänglich. Gezeigt werden hier Daten von Proben des Erzberges (Kalzitschiefer, Kalkmarmor und Phyllite). Ein Fokus bei den Messungen und Korrelationen wurde dabei auf die Anisotropie gelegt, die häufig vernachlässigt wird, aber von großer Auswirkung sein kann. Daten umfassen zum einen petrophysikalische Messungen, wie Porosität, Dichte und Kompressions- sowie Scherwellengeschwindigkeiten und zum anderen geotechnische, wie Abrasivitäten, statischen E-Modul und Druckfestigkeit. Zusätzlich wurden mineralogische Analysen durchgeführt, um die Zusammensetzung der Gesteine für eine Interpretation miteinfließen zu lassen. Gezeigt wird ein Teil der Daten und erste Korrelationen zwischen den Parametern. Die ersten Ergebnisse zeigen positive Trends. Für weitere detaillierte Aussagen müssen noch größerer Datenmengen gemessen werden.
\end{abstract}

Schlüsselwörter: Petrophysik, Geotechnik, Korrelationen, Mineralogie

Correlation of Petrophysical and Geomechanical Properties of Rocks from the Erzberg

Abstract: The correlation of geophysical/petrophysical parameters with geotechnical ones offers great added value for the planning and creation of underground infrastruc-

N. Gegenhuber ( $\triangle)$

Lehrstuhl für Subsurface Engineering,

Montanuniversität Leoben,

Erzherzog-Johann Straße 3,

8700 Leoben, Österreich

Nina.gegenhuber@unileoben.ac.at tures as well as geothermal and oil projects. Especially the interdisciplinary approach of geology, mineralogy, geophysics, and geotechnics can help with the challenges of the time in the future and is therefore inevitable. This contribution shows data from samples from the ore mountain (calcite slate, limestone marble, and phyllite). A focus in the measurements and correlations was placed on the anisotropy, which is often neglected but can have a major impact. Data include, on the one hand, petrophysical measurements, such as porosity, density and compression, and shear wave velocity, and, on the other hand, geotechnical measurements, such as abrasiveness, static modulus of elasticity, and compressive strength. In addition, mineralogical analyses were carried out to incorporate the composition of the rocks for an interpretation. Part of the data and initial correlations between the parameters are shown. The first results show positive trends. For more detailed statements, however, larger amounts of data still must be measured.

Keywords: Petrophysics, Geotechnics, Correlations, Mineralogy

\section{Einleitung}

Geophysik und als Teil davon die Petrophysik sind schon lange ein unersetzliches Gebiet in der Vorauserkundung und im Betrieb im Bereich der Erdölgewinnung. Ebenfalls in der Geothermie hat es seinen fixen Platz in der Vorauserkundung. Im Bereich der Geotechnik und des Tunnelbaus jedoch besteht hier noch Nachholbedarf oft auch durch Unwissenheit an den Möglichkeiten, die das Fachgebiet bieten kann.

Gerade die Petrophysik liefert im Labor, sowie auch bei Bohrlochmessungen, eine perfekte Synergie zur Geotechnik. Daher war es naheliegend, die Möglichkeit des Zentrum am Berg und die dabei gewonnen Proben aus dem Vortrieb für Messungen im Labor zu nutzen und daraus mögliche 


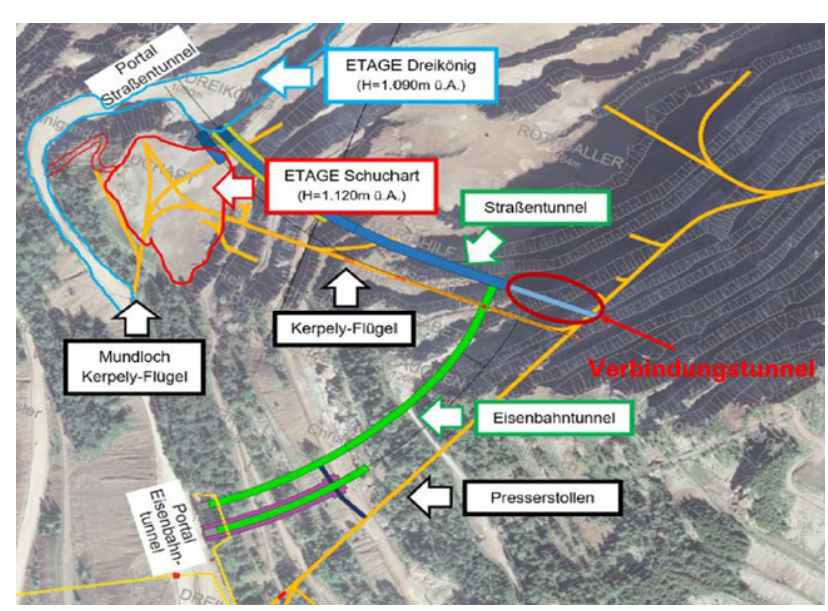

Abb. 1: Übersicht über das Zentrum am Berg, mit dem Verbindungstunnel, aus welchem die Proben stammen [6]

neue Korrelationen abzuleiten bzw. bestehende in einem ersten Ansatz zu bestätigen.

Eine Vielzahl an Artikel beschäftigen sich mit unterschiedlichsten Korrelationen, zum einen eher mit Fokus auf der Petrophysik, zum anderen mit dem Fokus auf den geotechnischen Parametern. Vernachlässigt wird dabei meist die Anisotropie. Die meisten Veröffentlichungen, die sich auf die Anisotropie von UCS konzentrieren, verwenden sie nie, um die Daten mit petrophysikalischen Daten zu korrelieren. Sie präsentieren Anisotropiedaten mit unterschiedlichem UCS in verschiedenen Richtungen und die Beschreibung dieser Effekte (z.B. [1-3] oder [4]). [5] maß UCS und Kompressionswellengeschwindigkeit in $15^{\circ}$ Schritten unterschiedlicher Richtung an Sandsteinproben, setzte diese jedoch nicht für eine Korrelation zueinander in Beziehung. Nur wenige bringen diese zusammen und inklu- dieren obendrauf noch mineralogische Einflüsse, welche sowohl petrophysikalische als auch geotechnische Eigenschaften beeinflussen. Nur ein multi-disziplinärer Ansatz kann hier in Zukunft ein neues Verständnis bzw. neue Korrelationen und Ansätze liefern und so optimale Ergebnisse in der Planung und Durchführung von Projekten bringen.

\section{Proben, Geologie und Messmethodik}

\subsection{Proben}

Probenmaterial für diese Studie entstammt dem Tunnelvortrieb im Verbindungstunnel des Zentrum am Berg (Abb. 1). Der Vortrieb erfolgte mittels Bohren und Sprengen. Die Bezeichnung der Proben definiert die Tunnelmeter (zw. 345 und $440 \mathrm{~m}$ ). Es wurden Phyllite, Kalksilikatschiefer und Marmor für die Messungen herangezogen. Um eine vollständige Beschreibung der Daten zu erzielen, wurden neben petrophysikalischen und geomechanischen Untersuchungen auch mineralogische und röntgendiffratometrische Messungen durchgeführt. Bei den Phylliten und den Schiefern wurde zusätzlich auf die Anisotropie Rücksicht genommen, da diese häufig vernachlässigt wird, aber eine große Rolle spielen kann.

\subsection{Geologie}

Der Erzberg ist Teil der Grauwackenzone bestehend aus paläozoischen Gesteinen. Nördlich der Grauwackenzone liegen die nördlichen Kalkalpen, im Süden wird sie von permomesozoischen Deckschickten getrennt [7]. Die nördliche Grauwackenzone (NGZ) erstreckt sich über ca. 450 km Län-
Abb. 2: Geologischer Überblick der Grauwackenzone (nach [8] verändert durch [9])

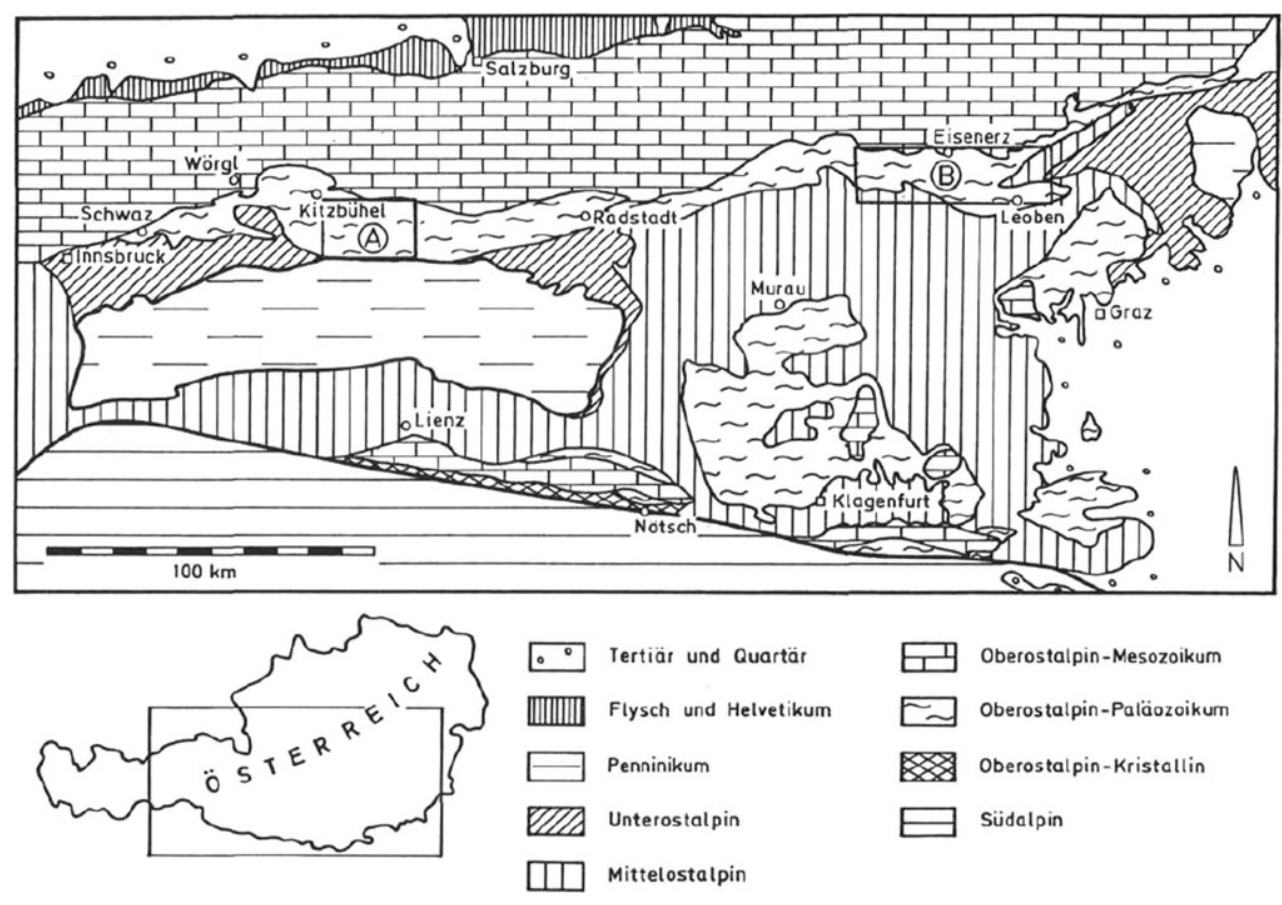




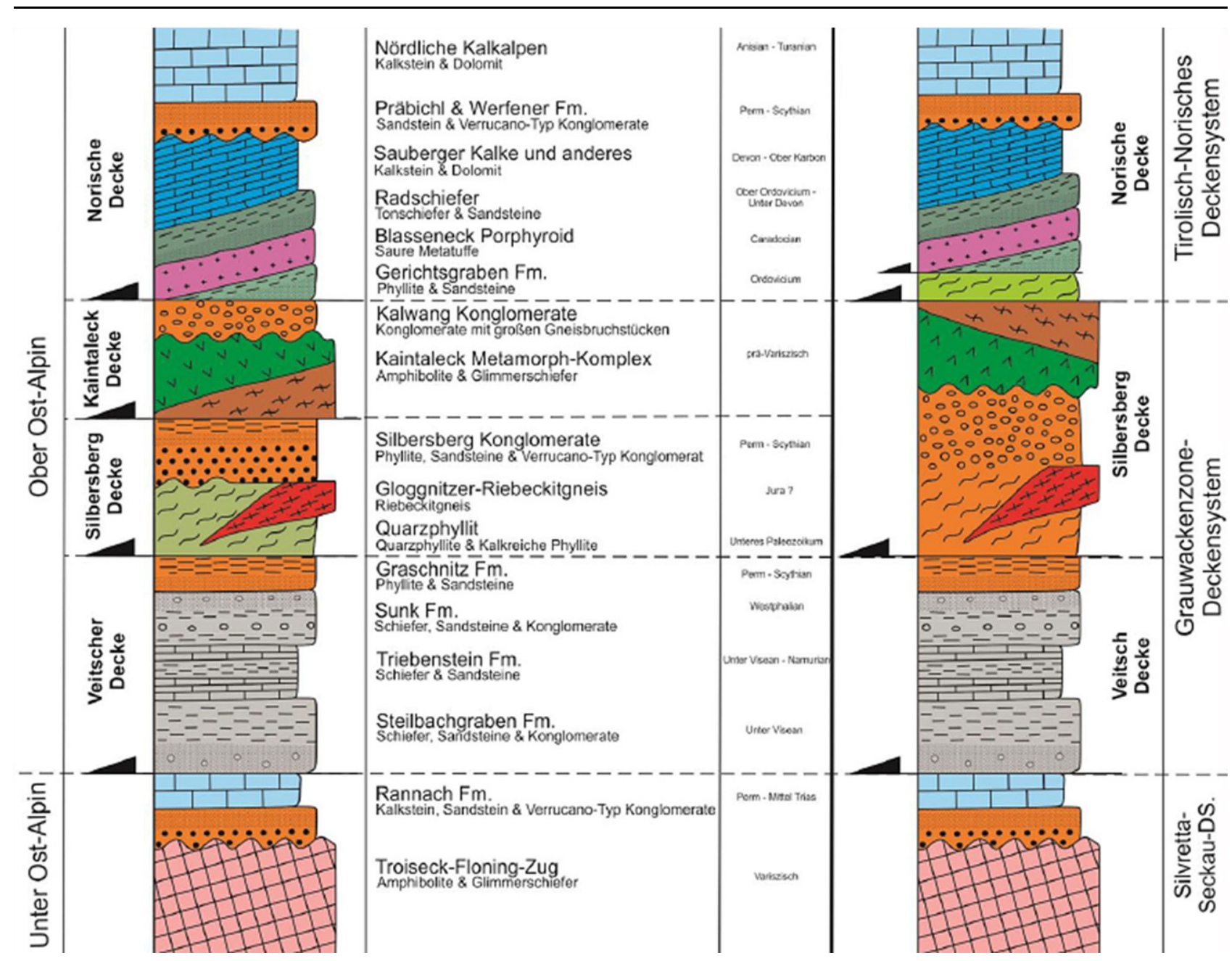

Abb. 3: Tektonische und lithostratigrafische Gliederung der Grauwackenzone. Links: nach [10] Rechts: nach [11] Verändert durch [12]
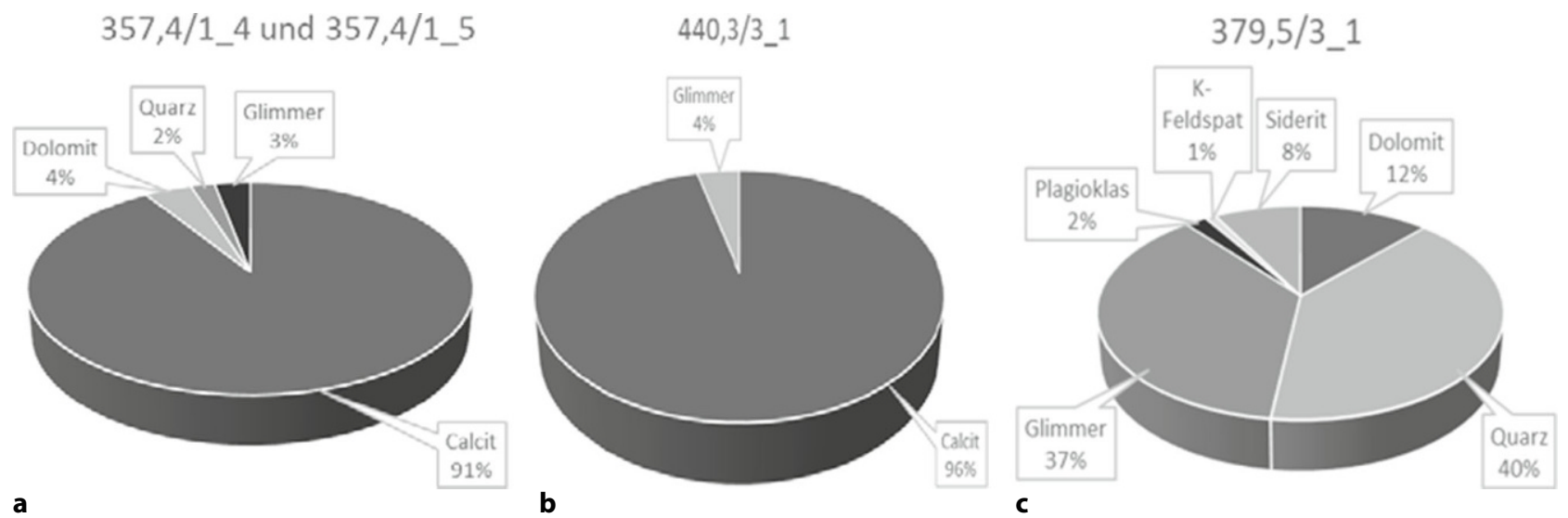

Abb. 4: Beispiele für je einen Gesteinstyp (von rechts nach links): Kalksilikatschiefer, Kalkmarmor, Phyllit

ge, von Schwaz in Tirol im Westen bis nach Ternitz im Osten (Abb. 2).

Die Grauwackenzone gliedert sich vom liegenden zum hangenden in folgende vier Decken (Abb. 4; [10]). Die Veitscher Decke, welche aus karbonen-permischen Gesteinen aufgebaut ist. Die Silbersberg Decke deren Hauptbestandteil die grünlichen Quarzit-Phyllite bilden. Die aus kristalli- nen Gesteinen aufgebaute Kaintaleck Decke und die Norische Decke, bestehend aus einer mächtigen Sequenz klastischer, vulkanischer und karbonatischer Formationen.

Nach [11] werden die Silbersberg- und Veitscher Decke zum Grauwackenzone-Deckensystem zusammengefasst. Dabei wird die von [10] beschriebene Kaintaleck Scholle mit der Silbersberg Decke zusammengelegt (Abb. 3). 


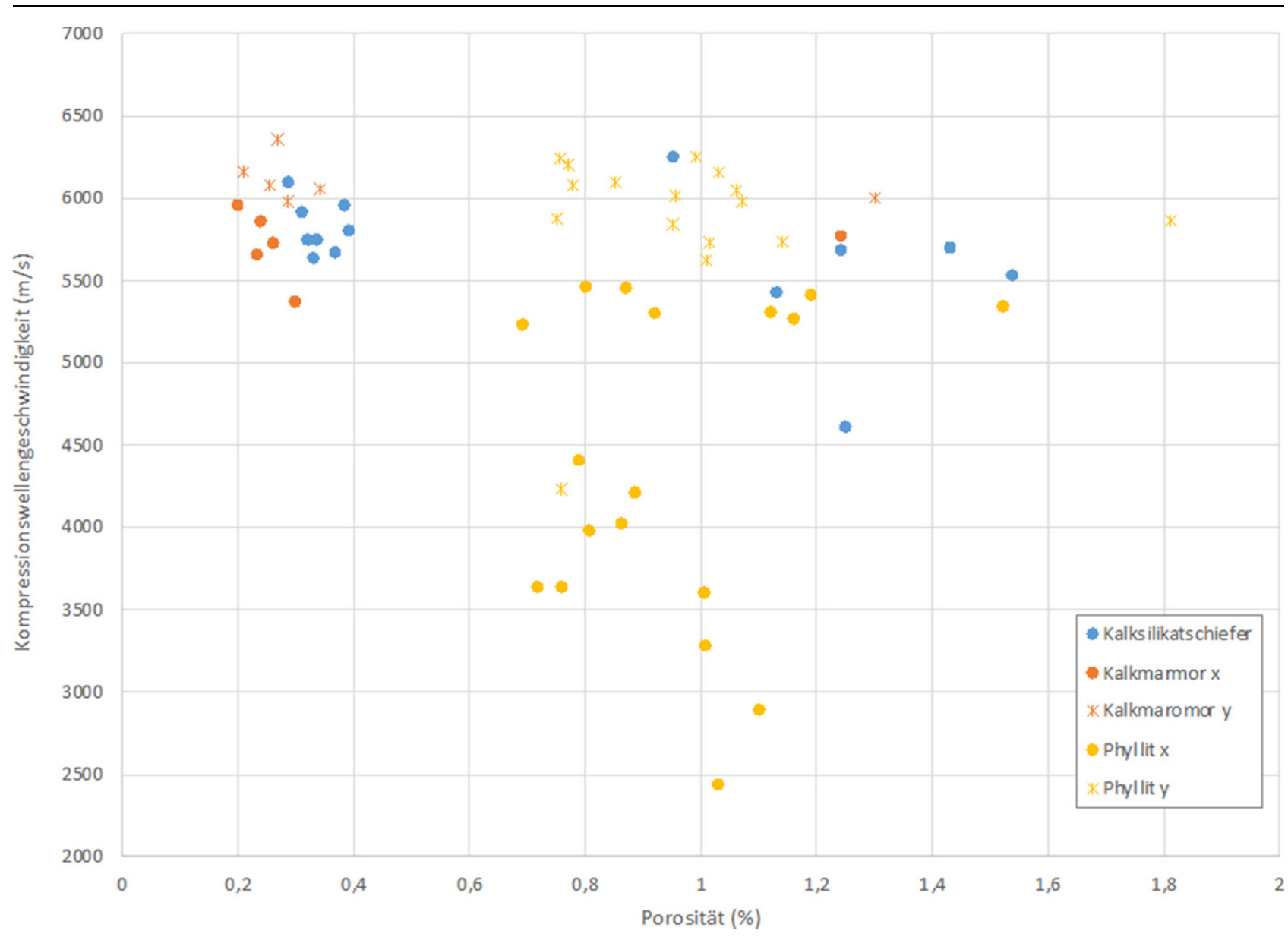

Abb. 5: Kompressionswellengeschwindigkeit gegen effektive Porosität, unterschieden werden die 3 Lithologien nach Farben, sowie die Richtung durch unterschiedliche Symbole

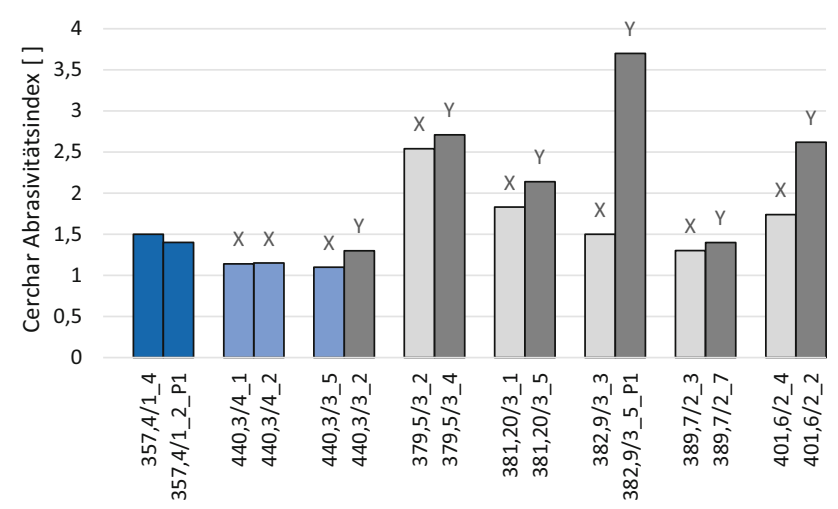

Abb. 6: Cerchar Index der einzelnen Proben, in hellblau der Kalksilikatschiefer, in dunkelblau der Marmor und in grau der Phyllit

\subsection{Messmethodik}

Die Proben wurde gebohrt und gesägt, um eine Planparallelität zu erzielen. Zum einen wurden Proben mit $5 \mathrm{~cm}$ Durchmesser und einer Länge von $10 \mathrm{~cm}$ gebohrt. Diese wurden neben den Kompressions $\left(v_{p}\right)$ - und Scherwellengeschwindigkeitsmessungen $\left(v_{s}\right)$ auch für die einaxialen Druckversuche herangezogen sowie für die $v_{p}$ und $v_{s}$ Messungen während des Druckversuchs [13]. $v_{p}$ und $v_{s}$ wurden mit ei- nem Ultraschallmessgerät erfasst [14]. Zum anderen wurden Proben mit $5 \mathrm{~cm}$ Durchmesser und $2,5 \mathrm{~cm}$ Höhe präpariert. An diesen Proben wurden Gesamt- und Korndichte sowie Porosität, $\mathrm{v}_{\mathrm{p}}$ und $\mathrm{v}_{\mathrm{s}}$ trocken und Wasser gesättigt und ein Cerchar Test durchgeführt. Alle Proben wurden bis zur Massenkonstanz bis zu $24 \mathrm{~h}$ bei $105 \mathrm{C}^{\circ}$ getrocknet.

Bei der Probenvorbereitung wurde auf Anisotropie geachtet und, wo eine solche makroskopisch erkennbar war, in zwei Richtungen gebohrt. Aus den Reststücken wurden zum einen Dünnschliffe hergestellt und zum anderen für den Los Angeles (LA) Test Material mit einer Korngröße von 14-12,5 mm, für den LCPC mit einer Korngröße von 6,3-4 mm und für die Röntgendiffraktometrie mit einer Korngröße $<1 \mathrm{~mm}$ aufgebrochen. Das gebrochene Material wurde ebenfalls nach der Herstellung bis zur Massenkonsistenz mit $105^{\circ} \mathrm{C}$ getrocknet.

Für die Gesamtdichte wurden die Proben jeweils dreimal in Länge und Durchmesser mit e vermessen und der Durchschnittswert ermittelt. Aus daraus berechnetem Volumen und bestimmter Masse berechnet sich die Gesamtdichte. Die Korndichte wurde mit einem Helium Pyknometer bestimmt. Aus Korndichte und Gesamtdichte ermittelt sich die effektive Porosität.

Der sogenannte Cerchar-Test (Centre d'Etudes et Recherches de Charbonnages de France) ist ein Versuch, bei dem 
Abb. 7: Korrelation der Parameter CAl und ABR sowie Korrelation der Parameter LA-Koeffizient und $B_{R}$, farbliche Unterscheidung wieder hinsichtlich der Gesteinstypen

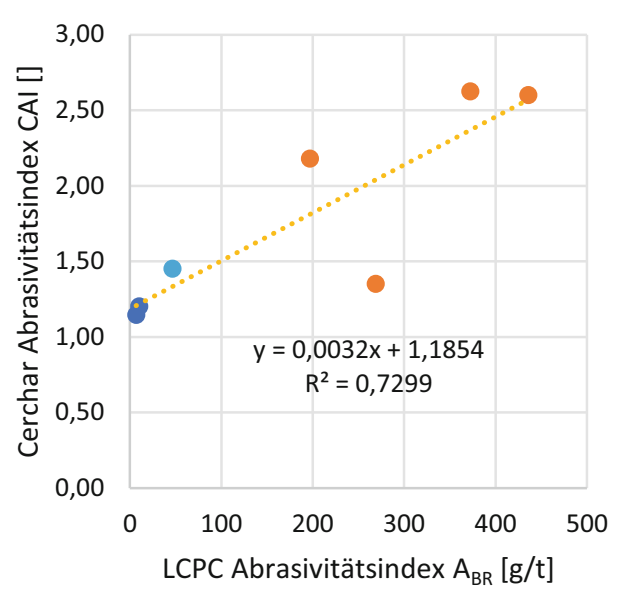

- Marmor

Kalkstein

a

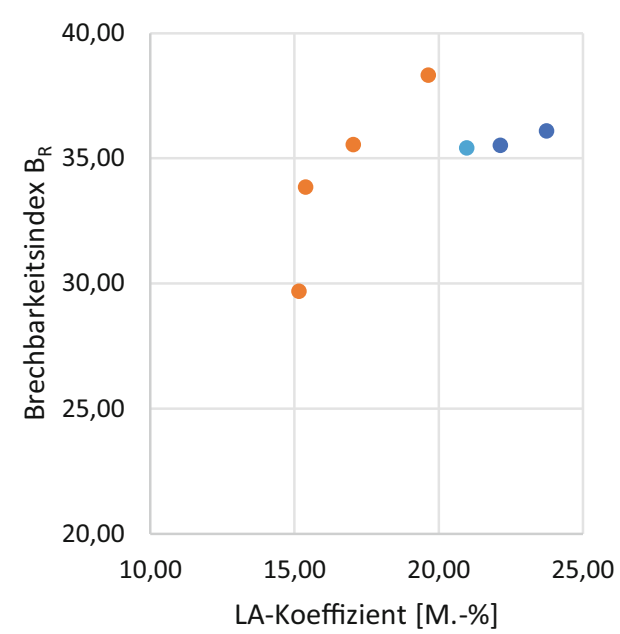

b Kalkstein - Marmor Phyllit
Abb. 8: Einaxiale Druckfestigkeit gegen Kompressionswellengeschwindigkeit, unterschieden werden nur die beiden Hauptrichtungen

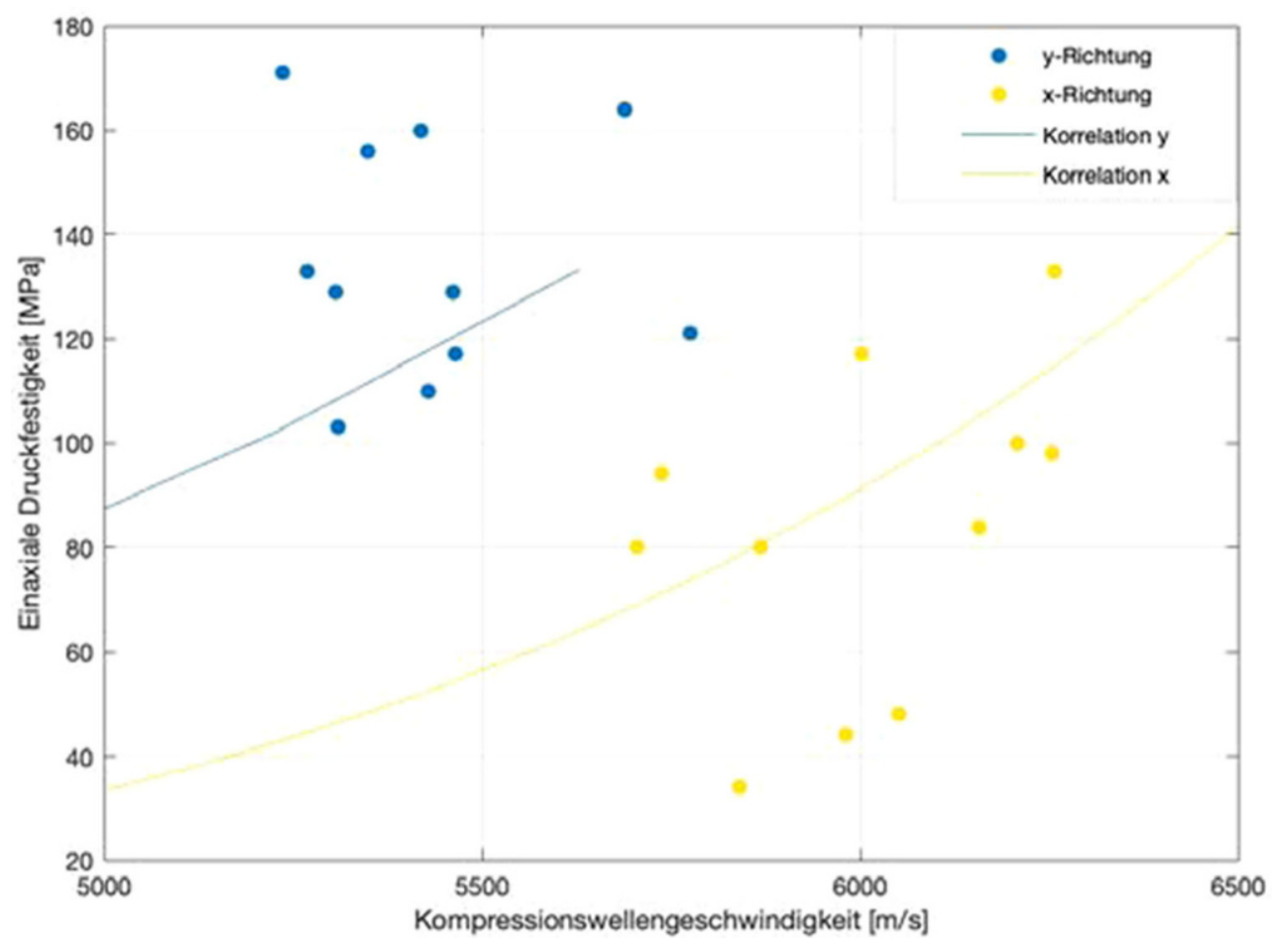

ein Prüfstift unter konstanter Auflast über eine definierte Prüfstrecke auf der Oberfläche eines handstückgroßen Probekörpers gezogen wird [15]. Für die Berechnung des sogenannten Cerchar Abrasivitätsindex (CAI) ist es notwendig, die Breite der am Prüfstift entstandenen kegelstumpfförmigen Verschleißrille zu messen. Für jede Probe sollten aufgrund der unterschiedlichen mineralogischen Zusammensetzung des Gesteins zwei bis fünf Einzelversuche durchgeführt werden, um einen aussagekräftigen Mittelwert bilden zu können [16]. Mit Hilfe des Cerchar-Tests können Festgesteine hinsichtlich ihrer Abrasivität untersucht und klassifiziert werden, um so den Verschleiß gebirgslösender Werk- zeuge (Bohrkrone, Disken, Meißel) prognostizieren zu können [15].

Der LCPC Abrasivitätsversuch (Laboratoire Central des Ponts et Chaussées) liefert zwei wichtige Parameter: Abrasivität und Brechbarkeit. Dieser Versuch wird mit einer Probenmenge von $500 \mathrm{~g} \pm 2 \mathrm{~g}$ gebrochenem Material durchgeführt. Die Probe wird in einen zylindrischen Behälter mit definierter Abmessung gefüllt und im Anschluss durch einen Drehflügel bei einer Umdrehungsgeschwindigkeit von $4500 \mathrm{U} / \mathrm{min} 5 \mathrm{~min}$ lang beansprucht.

Mit Hilfe des Los Angeles Tests wird der Widerstand gegen Zertrümmerung geprüft. Dieser Widerstand stellt das wesentliche Prüfmerkmal für die Beschreibung der Festig- 


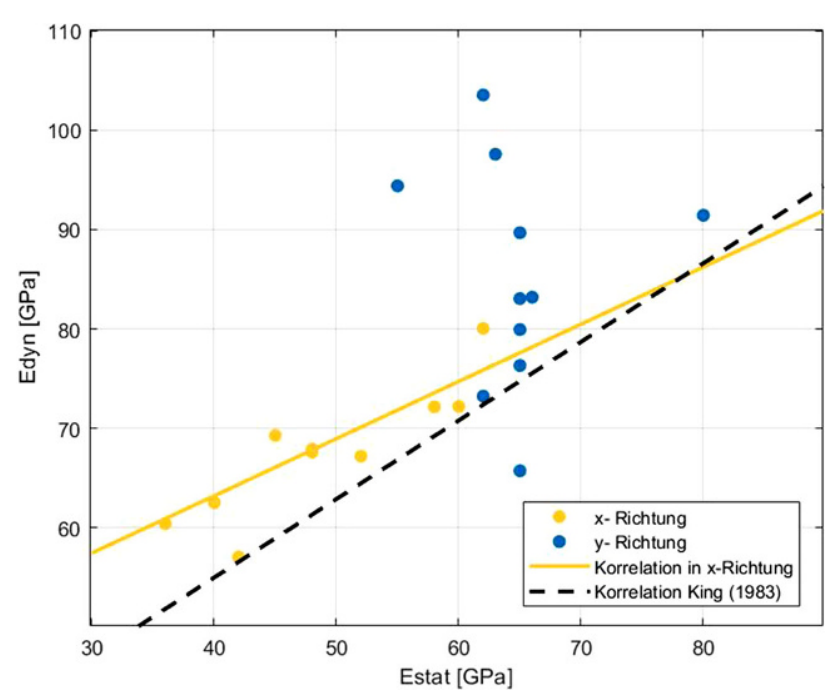

Abb. 9: E dynamisch versus E statisch, Unterscheidung nach Richtung

keit von groben Gesteinskörnungen im Straßenbau dar und wird über den Los-Angeles-Koeffizienten ausgedrückt.

\section{Ergebnisse}

Für die Messergebnisse werden die Daten zum einen hinsichtlich der bereits bekannten Zusammenhänge wie $v_{p}$ gegen Porosität analysiert, wobei zusätzlich immer ein Fokus auf die Anisotropie und den Lithologie Einfluss gelegt wird. Zum anderen zeigt der zweite Teil neue Versuche, Parameter miteinander zu korrelieren. Es werden hier nur ausgwählte Abbildungen der Ergebnisse präsentiert.

Abb. 4 zeigt die Ergebnisse der Röntgendiffraktometrie der drei Gesteinstypen, einzelner ausgewählter Proben, um einen ersten Eindruck der mineralogischen Zusammensetzung zu zeigen. Der Kalksilikatschiefer besteht zum Großteil aus Kalzit mit Nebengemengteilen von Dolomit, Quarz und Glimmer. Der Kalkmarmor besteht zum Hauptteil aus Kalzit mit nur geringen Nebenanteilen. Der Phyllit variiert mit Quarzanteil zwischen 38 und $42 \%$ als Hauptanteil. Daneben treten Glimmer, Dolomit, Siderit, Plagioklas und zum Teil Kalifeldspat in unterschiedlichen Anteilen auf.

Abb. 5 zeigt als Übersicht die Kompressionswellengeschwindigkeit gegen die Porosität. Farblich unterschieden werden die drei Gesteinstypen (und somit der Einfluss der Mineralogie/Lithologie) sowie zusätzlich die gebohrte Richtung jener, wo makroskopisch eine Unterscheidung möglich war. Gut erkennbar ist der generelle Trend zur Abnahme der $v_{p}$ mit Zunahme der Porosität. Des weiteren erkennbar sind der Kalkmarmor und der Kalksilikatschiefer, der niedrigere Porositäten und höhere Geschwindigkeiten zeigt. Der Phyllit zeigt eine breite Streuung vor allem in der $x$-Richtung. Dies ist auf Inhomogenitäten zurückzuführen, da die Schichten nicht gleichmäßig angeordnet sind, sondern häufig in sich noch kleine Verformungen zeigen. Dies wird sich in auch in Folge bei den Korrelationen der E-Module zeigen.

Die Richtungsabhängigkeit die man bei den Kompressionswellengeschwindigkeiten sieht, spielgelt sich auch in den Cerchar Messungen (Abb. 6) wieder. Die Phyllite zei- gen durch den höheren Quarzgehalt eine höhere Abrasivität und zusätzlich liegen die Werte der y-Richtung etwas höher. Es wurden nur von ausgewählten Proben Cerchar Messungen durchgeführt. Eine weitere Messserie wird hier vermutlich noch aussagekräftigere Daten liefern. Gerade die Korrelation Cerchar Index mit $v_{p}$ könnte in der Praxis hilfreich sein. Die Phyllite zum Beispiel zeigen höhere CAI und niedrigere $\mathrm{v}_{\mathrm{p}}$ Werte.

Die von [17] beobachtete Korrelation zeigt sich ansatzweise auch für diese Ergebnisse. Dabei deutet sich ein Trend einerseits für die Messpunkte des Phyllits und andererseits für die Resultate des Marmors und Kalksteins an. Zusätzliche Versuche sind notwendig, um die Punktdichte zu erhöhen. Abb. 7 zeigt eine Gegnüberstellung des CAI Wertes aus dem Cerchar Versuch mit dem $A_{B R}$ Wert des LCPC Versuches. Der geplottete Trend zeigt eine lineare Zunahme des CAI Wertes mit steigendem $A_{B R}$ Wert. Die Resultate aus den Versuchen liefern dabei ein gutes erstes Verhältnis.

Druckfestigkeiten aus einaxialen Druckversuchen und der daraus resultierende E-Modul statisch und den während des Druckversuches bestimmten $v_{p}$ (daraus abgeleitet E-Modul dynamisch) wurden nur an ausgewählten Proben gemessen.

Abb. 8 und 9 zeigen die Ergebnisse und daraus resultierende Korrelationen für weitere Anwendungen. Die Korrelationen wurden mit Modellrechnungen durchgeführt. Details findet man hierzu in [18]. Diese Korrelationen können in Folge auf Bohrlochdaten angewandt werden, wenn ein Sonic Log und damit $v_{p}$ vorhanden ist und die Lithologie bekannt ist.

\section{Zusammenfassung und Ausblick}

Erste Ergebnisse zeigen vielversprechende Korrelationen für weitere Anwendungen. Für die Entwicklung weiterer aussagekräftigere Zusammenhänge müssen weitere Messungen durchgeführt werden, um eine bessere Datenbasis und vor allem Datendichte zu haben. Korrelationen zwischen geomechanischen und petrophysikalischen/ geophysikalischen Eigenschaften sind nicht nur im Bergund Tunnelbau, sondern auch in der Erdölindustrie, der Geothermie und hydrogeologischen Feldern von großem Interesse.

Dieser Beitrag soll einen ersten Überblick über die bisherigen Messergebnisse liefern. Dafür werden metamorphe Proben vom Erzberg in Österreich verwendet. Ein Hauptproblem in y-Richtung besteht darin, dass die Phyllite in sich gefaltet sind und manchmal keine richtigen Schichten zu sehen sind, was zu einigen Inhomogenitäten führt und die Korrelationen erschwert. Wichtig ist, dass anisotrope Effekte zwar stark sichtbar sind und zeigen, dass sie unbedingt berücksichtigt werden müssen. Die Korrelation zwischen UCS und $v_{p}$ zeigt gute Ergebnisse. Die resultierenden Gleichungen können außerdem auf Bohrlochdaten angewendet werden, um direkt vor Ort geomechanische Eigenschaften abzuleiten. Die untersuchten Gesteinstypen sind anisotrop und in verschiedenen Skalen inhomogen. Wir können daraus schließen, dass die einzelnen Gesteinskomponenten und ihre Ausrichtung und Form dynamische und 
statische Messungen unterschiedlich beeinflussen; dieser Unterschied ist - in unserem Fall - für die y-Richtung sehr stark. Daher ist es wiederum wichtig, anisotrope Effekte für Korrelationen zwischen petrophysikalischen und geotechnischen Eigenschaften zu berücksichtigen. Dieser interdisziplinäre Ansatz der einzelnen Fachbereiche ist von großer Bedeutung für zukünftige Projekte.

Funding. Open access funding provided by Montanuniversität Leoben.

Open Access Dieser Artikel wird unter der Creative Commons Namensnennung 4.0 International Lizenz veröffentlicht, welche die Nutzung, Vervielfältigung, Bearbeitung, Verbreitung und Wiedergabe in jeglichem Medium und Format erlaubt, sofern Sie den/die ursprünglichen Autor(en) und die Quelle ordnungsgemäß nennen, einen Link zur Creative Commons Lizenz beifügen und angeben, ob Änderungen vorgenommen wurden.

Die in diesem Artikel enthaltenen Bilder und sonstiges Drittmaterial unterliegen ebenfalls der genannten Creative Commons Lizenz, sofern sich aus der Abbildungslegende nichts anderes ergibt. Sofern das betreffende Material nicht unter der genannten Creative Commons Lizenz steht und die betreffende Handlung nicht nach gesetzlichen Vorschriften erlaubt ist, ist für die oben aufgeführten Weiterverwendungen des Materials die Einwilligung des jeweiligen Rechteinhabers einzuholen.

Weitere Details zur Lizenz entnehmen Sie bitte der Lizenzinformation auf http://creativecommons.org/licenses/by/4.0/deed.de.

\section{Literatur}

1. Nasseri, M.H.B.; Rao, K.S.; Ramamurthy, T.: Anisotropic strength and deformational behaviour of Himalayan schists, International Journal of Rock Mechanics and Mining Sciences, 40 (2003), S. 3-23, https://doi.org/10.1016/S1365-1609(02)0010-X

2. Karakul, H.; Ulusay, R.; Isik, N.S.: Empirical models and numerical analysis for assessing strength anisotropy based on block punch index and uniaxial compression tests, International Journal of Rock Mechanics and Mining Sciences, 47 (2008), S. 657-665, https://doi. org/10.1016/j.ijrmms.2010.03.006

3. Saroglou, H.; Marinos, P.; Tsiambaos, G.: The anisotropic nature of selected metamorphic rocks from Greece, The Journal of The South African Institute of Mining and Metallurgy, 2004, S. 217-222

4. Bhuiyan, M.H.; Holt, R.M.; Fjaer, E.: Anisotropic parameters of dry and saturated sand under stress, SEG Annual Meeting, Houston, 2013, S. 2836-2840

5. Al-Harthi, A.A.: Effect of planar structures on the anisotropy of Ranyah sandstone, Saudi Arabia, Engineering Geology, 50 (1998), 49-57, doi.org/10.1016/S0013-7952(97)00081-1
6. Laabmayr IL - Ingenieurbüro Laabmayr \& Partner ZT GesmbH: U-Bahn Trainings- und Ausbildungszentrum. U-Bahn-Station Dreikönig, 2019

7. Schönlaub, H.P.: Der Ost Abschnitt Der Grauwackenzone - Ein Überblick. In: Geologische Bundesanstalt (Hg.): Arbeitstagung der Geologischen Bundesanstalt, 2019, S. 63-70

8. Bögel, H.; Schmidt, K.: Kleine Geologie der Ostalpen. Allgemein verständliche Einführung in den Bau der Ostalpen unter Berücksichtigung der angrenzenden Südalpen, 1967

9. Schlaegel-Blaut, P. (Hg.): Der basische Magmatismus der Nördlichen Grauwackenzone, Oberostalpines Paläozoikum, 1990, Zugl.: München, Univ., Diss.: 1989: Abhandlungen der Geologischen Bundesanstalt, Bd. 43, Wien: Geologische Bundesanstalt

10. Neubauer, F.; Handler, R.; Hermann, S.; Paulus, G.: Revised Lithostratigraphy and Structure of the Eastern Graywacke Zone (Eastern Alps), Austrian Journal of Earth Sciences, 86 (1993), S. 61-74

11. Schuster, R.: Grauwackenzone und Veitsch-Silbersberg-Deckensystem. (2015), In: Schuster, R., Ilickovic, T. (Hg.): Arbeitstagung 2015 der Geologischen Bundesanstalt Geologie der Kartenblätter GK50 ÖK 103 Kindberg und ÖK 135 Birkfeld. Mitterdorf im Mürztal 21.-25. September 2015. 2. korrigierte Auflage, S. 38-43. Wien: Geologische Bundesanstalt.

12. Haller, D.: Polyphase Deformation und Deckenstapelung in der östlichen Grauwackenzone, Masterarbeit, TU Graz, 2016

13. Pittino, G.; Gegenhuber, N.; Reiter, F.; Fröhlich, R.: Axiale Prüfkörperdurchschallung während einaxialer Druckversuche, Berg- und Hüttenmännische Monatshefte, 160 (2015), Nr. 12, S. 565-571, https:// doi.org/10.1007/s00501-015-0423-9

14. Gegenhuber, N.; Steiner-Luckabauer, C.: Vp/Vs Automatic Picking of Ultrasonic Measurements and their Correlation of Petrographic Coded Carbonates from Austria, In: 74th EAGE Conference and Exhibition incorporating EUROPEC 2012: Proceedings: EAGE Publications BVNetherlands, 2012

15. Käsling, H.; Plinninger, R.J.: Bestimmung der Abrasivität von Gesteinen mit dem CERCHAR-Versuch. Bautechnik, 93 (2016), S. 409-415

16. Plinninger, R.J.; Restner, U.: Abrasiveness Testing, Quo Vadis?-A Commented Overview of Abrasiveness Testing Methods. Geomechanik und Tunnelbau, 1 (2008), S. 61-70

17. Resch, D.: Verwendung von Tunnelausbruchmaterial - Entscheidungsgrundlagen, Dissertation, Universität bitte ergänzen Montanuniversität Leoben, 2012

18. Gegenhuber, N.; Krueger M.: Linking static and dynamic mechanical properties for metamorphic rocks from Austria including their anisotropic effect, Acta Geophysica, 69, S. 539-546 Jahrgang bitte ergänzen (2021), published online, https://doi.org/10.1007/s11600-02100566-w

Hinweis des Verlags. Der Verlag bleibt in Hinblick auf geografische Zuordnungen und Gebietsbezeichnungen in veröffentlichten Karten und Institutsadressen neutral. 